\title{
White matter changes and late-life depressive
}

\section{symptoms}

\author{
Longitudinal study
}

\section{A. TEODORCZUK, J. T. O'BRIEN, M. J. FIRBANK, L. PANTONI, A. POGGESI, T. ERKINJUNTTI, A. WALLIN, L.-O. WAHLUND, A. GOUW, G. WALDEMAR, \\ R. SCHMIDT, J. M. FERRO, H. CHABRIAT, H. BÄZNER and D. INZITARI on behalf of the LADIS Group}

Background Evidence from crosssectional studies suggests a link between cerebral age-related white matter changes and depressive symptoms in older people, although the temporal association remains unclear.

Aims To investigate age-related white matter changes on magnetic resonance imaging (MRI) as an independent predictor of depressive symptoms at I year after controlling for known confounders.

Method In a pan-European multicentre study of 639 older adults without significant disability, MRI white matter changes and demographic and clinical variables, including cognitive scores, quality of life, disability and depressive symptoms, were assessed at baseline. Clinical assessments were repeated at I year.

\section{Results Using logistic regression} analysis, severity of white matter changes was shown to independently and significantly predict depressive symptoms at I year after controlling for baseline depressive symptoms, quality of life and worsening disability $(P<0.0 \mathrm{I})$.

Conclusions White matter changes pre-date and are associated with the development of depressive symptoms. This has implications for treatment and prevention of depression in later life.

\section{Declaration of interest None.}

Funding detailed in Acknowledgements.
Cerebral white matter changes are strongly associated with depressive symptoms in older people (de Groot et al, 2000). However, the direction of causation remains unclear because most studies are crosssectional (O'Brien et al, 1996; Hickie et al, 1997; Steffens et al, 1999) and the possibility remains that both white matter changes and depressive symptoms are manifestations of a common pathological pathway, or that depressive symptoms pre-date the development of white matter changes.

The LADIS (Leukoaraiosis and Disability in the Elderly) Study is a large multicentre pan-European longitudinal study of older adults without significant disability which was established to investigate the relationship between white matter changes on magnetic resonance imaging (MRI) and subsequent development of disability, cognitive impairment and depressive symptoms. We have previously reported cross-sectional associations between white matter changes and depressive symptoms in this sample (Firbank et al, 2005; O'Brien et al, 2006). In this report we examine white matter changes as an independent predictor of future depressive symptoms. We hypothesised that the severity of white matter changes at baseline would be associated with the development of depressive symptoms at 1 year follow-up, irrespective of known confounding variables.

\section{METHOD}

\section{Recruitment}

Participants were recruited from the 11 European centres participating in the LADIS Study (Amsterdam, Copenhagen, Florence, Graz, Gothenburg, Helsinki, Huddinge, Lisbon, Mannheim, Newcastle upon Tyne and Paris). Full details of the study design have been reported previously (Pantoni et al, 2005).

In brief, inclusion criteria were: (a) between 65 and 84 years; (b) living in the community; (c) no or mild disability (only one item compromised) as assessed by the Instrumental Activities of Daily Living scale (IADL; Lawton \& Brody, 1969); (d) the presence of an informant; (e) any degree of age-related white matter changes on MRI scan according to a revised version of the scale of Fazekas et al (1987).

Exclusion criteria were: (a) the presence of severe illnesses (cardiac, hepatic or renal failure, neoplastic or other relevant systemic disease) which would increase the likelihood of drop-out; (b) severe unrelated neurological diseases; (c) leucoencephalopathies revealed by brain imaging that were of non-vascular origin (immunological, demyelinating, metabolic, toxic or infectious); (e) severe psychiatric disorders; (f) refusal or inability to give informed consent; (g) refusal or inability to undergo cranial MRI scanning.

There were 639 people who fulfilled the criteria and were recruited between July 2001 and January 2003. Most were recruited after presentation to centres with mild cognitive disturbances $(n=168)$, gait disturbances $(n=28)$, psychiatric complaints $(n=13)$, other neurological disturbances $(n=129)$, or minor stroke $(n=122)$. Other participants included those in whom white matter changes were incidentally found on computed tomography or MRI performed in other clinical settings $(n=107)$ and controls from other studies with brain white matter changes $(n=72)$. The total number of participants referred from each centre and the reasons for referral are given in Table DS1 of the data supplement to the online version of this paper. All participants gave informed consent.

\section{Assessment}

All participants had a comprehensive baseline demographic assessment by trained personnel. Information was collected on age, gender, education, occupational status, living conditions, previous medical conditions, including stroke (as defined according to the World Health Organization; Hatano, 1976) and hypertension (Chalmers et al, 1999), prescribed medication, lifestyle (alcohol and smoking) and vascular risk factors.

Further baseline clinical assessments included the following.

(a) Mini-Mental State Examination (MMSE) was used to assess cognition (Folstein et al, 1975). 
(b) Functional status in terms of disability was measured by the IADL. This is a self-reported scale developed to monitor function and independent living among older adults and measures a broad set of daily activities, including shopping for personal items, preparing meals, performing housework and managing personal finances.

(c) Health-related quality of life (QoL) was measured using the self-reported EuroQoL 5D (Euro-QoL Group, 1990). The participant indicates their current health state where 100 indicates best imaginable health and 0 the worst.

(d) Depressive symptoms were assessed by the self-completed 15-item Geriatric Depression Scale (GDS), which was developed as a screening instrument for depressive symptoms in elderly populations (Yesavage, 1988). The maximum possible score is 15 .

(e) A history of depression was recorded, along with the date of any incident depression. History of depression was defined as the presence of a depressive episode requiring treatment or hospital admission. Incident depression was defined as any depressive episode requiring treatment or hospital admission over the study year. Both history and incident depression were obtained through interview of the participant and evaluation of the case notes.

(f) Standard neurological and cardiovascular examination was performed and included blood pressure measurements.

Participants were re-evaluated at 1 year and clinical assessments were repeated. To increase reliability, investigators were issued with a specifically designed handbook which contained guidelines for applying tools.

\section{Magnetic resonance imaging}

Participants had a baseline MRI scan at their respective centres. All centres used MRI scanners with a field strength of 1.5 T, except for one which had a $0.5 \mathrm{~T}$ system. A standard protocol was used (Pantoni et al, 2005). For the white matter rating, a FLAIR sequence was acquired with the following parameters: $250 \mathrm{~mm}$ field of vision; $256 \times 256$ or $256 \times 192$ matrix; $5 \mathrm{~mm}$ slice thickness; $0.5 \mathrm{~mm}$ slice gap; $19-28$ slices; time to echo $100-140 \mathrm{~ms}$; time to repetition 6000-10000 ms; inversion time 2000 $2500 \mathrm{~ms}$, and echoes per shot 7-24.
Volumetric analysis of age-related white matter changes was performed on a Sparc 5 workstation (Sun, Palo Alto, California, USA; van Straaten et al, 2006). Lesions were marked using a 'seed' and local thresholding was performed using home-developed software (Show Images, version 3.6.1 using a Canay filter) on each slice. When necessary, borders could be adjusted by the operator by changing thresholds for upper and lower intensity values. If all lesions are delineated, the program calculates the total surface of the outlined area. By multiplying with the interslice distance, total volume of agerelated white matter changes is established (Gouw et al, 2006).

\section{Statistical analysis}

Data were collected in each centre and entered into a central electronic database on a specifically developed website (http:// www.unifi.it/LADIS). In a communitydwelling population it is normal for depression rating scales to be heavily skewed towards low values. Hence for analysis we divided our baseline and 1-year data into quintiles, using the same GDS range for each quintile as in our previous study (O'Brien et al, 2006). For the volume of age-related white matter changes we used a logarithmic transformation to produce normally distributed data.

Univariate analysis was used to examine correlations between different variables and depressive symptoms at 1 year. As depressive symptoms (as measured by GDS) are on an ordinal scale, we used Spearman's rank order correlation coefficient $\left(r_{2}\right)$ to determine the correlation between depressive symptoms at 1 year, the dependent variable and various independent demographic, clinical and MRI variables. Baseline independent variables included age, gender, baseline depressive symptoms (GDS), educational level, smoking status, MMSE score, stroke, hypertension, QoL and log total volume of white matter changes. Incident stroke (a new stroke over the study year) and worsening disability (an IADL score at 1 year less than at baseline) were included as further independent variables.

Multivariate analysis used ordinal logistic regression to determine predictors of the quintile of depression scale score at 1 year. The variables chosen were those which were significant in the univariate analysis and included GDS baseline quintile, worsening disability, QoL, MMSE score, years of education, incident stroke and total volume of white matter changes. We constructed a further binary logistic regression to compare predictors of a depressive episode over the year using history of depression (instead of GDS quintile) together with the other significant independent variables as predictors.

Values of $P<0.05$ were considered statistically significant.

\section{RESULTS}

\section{Participants}

Of the original cohort of 639 participants there were missing baseline data for MMSE score in 1 , presence of stroke in 1 , years of education in 1 , hypertension in 2 , total volume of white matter changes in 21 and QoL rating in 6. From the remaining 607, 6 people died during the year and 12 dropped out, leaving 589 participants at 1-year follow-up. There were missing follow-up data for a further 63 participants. Of these some had multiple variables missing; GDS score in 54; IADL score in 53; QoL score in 62; MMSE score in 57; and history of depression in 50. Thus there were 526 participants in the final analysis. To determine if there had been any effect of selective drop-out, we compared the baseline GDS, total volume of white matter changes, QoL and MMSE scores between those who did and did not have follow-up data. The baseline MMSE score was significantly lower in those without (mean 26.2, s.d.=2.5) compared with those with followup data (mean 27.6, s.d.=2.3). There were no significant differences in the other examined variables.

As expected for a community population, the group GDS score remained stable over time. The median GDS score was 2 at baseline and at 1 year (Wilcoxon signed-rank $Z=-1.77, P=0.077)$. At baseline, 141 people had a history of depression for which medical help had been sought. Over the year, 85 people had an episode of depression. The number of people in each quintile of GDS at baseline and 1 year is given in Table DS2 of the data supplement to the online version of this paper.

Table 1 shows the demographic, clinical and MRI characteristics of the participants at baseline. Over the year, 14 participants had an incident stroke and 56 had worsening disability. 
Table I Baseline characteristics of the $\mathbf{5 2 6}$ participants

\begin{tabular}{lc}
\hline Characteristic & \\
\hline $\begin{array}{l}\text { Age, years: mean (s.d.) } \\
\text { Gender }\end{array}$ & $73.9(5.0)$ \\
$\quad$ Female & 291 \\
$\quad$ Male & 235 \\
GDS score: median (range) & $2(0-14)$ \\
History of depression, $n$ (\%) & 141 (27) \\
History of hypertension, $n$ (\%) & $376(71)$ \\
History of stroke, $n$ (\%) & $152(29)$ \\
Years of education: mean (s.d.) & $9.8(3.9)$ \\
MMSE score: mean (s.d.) & $27.6(2.3)$ \\
QoL score: mean (s.d.) & $72.6(17.2)$ \\
Total volume of white matter & \\
changes, ml: median (range) & I3.5 (0.7-129)
\end{tabular}

GDS, Geriatric Depression Scale; MMSE, Mini-Mental State Examination; QoL, Quality of Life.

Table 2 Univariate analysis of predictors with quintile of GDS score at I year

\begin{tabular}{lrr}
\hline & \multicolumn{1}{c}{$r_{\mathrm{s}}$} & \multicolumn{1}{c}{$P$} \\
\hline Age in years at inclusion & 0.05 & 0.237 \\
Gender & -0.06 & 0.165 \\
Baseline GDS quintile & 0.62 & $<0.001$ \\
QoL score & -0.43 & $<0.001$ \\
Worsening IADL & 0.25 & 0.001 \\
MMSE score & -0.11 & 0.010 \\
Years of education & -0.20 & $<0.001$ \\
Baseline history of & & \\
stroke & 0.08 & 0.052 \\
Incident stroke & 0.13 & 0.030 \\
Smoking (never v. & & \\
current/past) & 0.00 & 0.909 \\
Arterial hypertension & 0.06 & 0.165 \\
Log total volume of & & \\
white matter changes & 0.21 & $<0.001$ \\
\hline GDS, Geriatric Depression Scale & $M M S E M i M e n t a l$
\end{tabular}

GDS, Geriatric Depression Scale; MMSE, Mini-Menta State Exam; IADL, Instrumental Activities of Daily Living; QoL, Quality of Life.

\section{Univariate analysis}

Table 2 shows the results of the univariate analysis. As expected, the GDS quintile at baseline, incident stroke and worsening disability were significantly positively correlated with GDS quintile at 1 year. MMSE score, number of years education and QoL score were significantly negatively correlated with GDS quintile at 1 year.

Log total volume of white matter changes was also significantly positively

Table 3 Ordinal logistic regression of predictors of quintile GDS at I year

\begin{tabular}{lcc}
\hline Independent variable & Odds ratio $(95 \% \mathrm{Cl})$ & $P$ \\
\hline Baseline GDS quintile & $2.66(2.45-2.89)$ & $<0.00 I$ \\
QoL & $0.98(0.98-0.99)$ & $0.00 I$ \\
Worsening IADL & $3.72(2.78-4.99)$ & $<0.001$ \\
MMSE score & $1.02(0.98-1.06)$ & 0.6 \\
Years of education & $0.96(0.94-0.98)$ & 0.1 \\
Incident stroke & $1.37(0.80-2.36)$ & 0.6 \\
Log total volume of white matter changes & $I .65(1.37-1.98)$ & 0.007 \\
\hline
\end{tabular}

GDS, Geriatric Depression Scale; MMSE, Mini-Mental State Exam; IADL, Instrumental Activities of Daily Living; QoL, Quality of Life.

Table 4 Binary logistic regression of predictors of occurrence of depression over the year

\begin{tabular}{lcc}
\hline Independent variable & Odds ratio $(95 \% \mathrm{Cl})$ & $P$ \\
\hline History of depression & $8.93(6.78-11.8)$ & $<0.00 I$ \\
QoL & $0.98(0.98-0.99)$ & 0.046 \\
Worsening IADL & $2.16(1.47-3.17)$ & 0.045 \\
MMSE score & $0.94(0.89-0.99)$ & 0.3 \\
Years of education & $1.03(0.99-1.07)$ & 0.5 \\
Incident stroke & $1.07(0.49-2.36)$ & 0.9 \\
Log total volume of white matter changes & $1.63(1.20-2.20)$ & 0.1
\end{tabular}

GDS, Geriatric Depression Scale; MMSE, Mini-Mental State Examination; IADL, Instrumental Activities of Daily Living; QoL, Quality of Life.

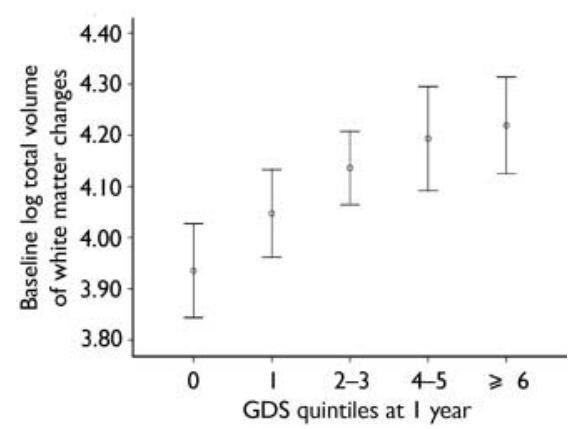

Fig. I Relationship between baseline log total volume of white matter changes and Geriatric Depression Scale (GDS) quintile at I year. Standard error bars are shown.

correlated with GDS quintile at 1 year (Fig. 1).

\section{Multiple regression analysis}

Table 3 shows logistic ordinal regression with GDS quintile at 1 year as the dependent variable and baseline GDS quintile, QoL score, worsening IADL, MMSE score, years of education, incident stroke and $\log$ total volume of white matter changes as independent variables. Baseline GDS quintile, QoL, worsening IADL and log total volume of white matter changes were significantly and independently associated with GDS quintile at 1 year.

To control for the effect of MRI centre as a potential confounder we constructed a further logistic regression model including MRI centre as an additional independent variable, and found that baseline log total volume of white matter changes still significantly and independently predicted GDS quintile at 1 year $(P=0.046)$.

Table 4 shows a further binary regression with occurrence of depression over the year as the dependent variable and baseline history of depression, QoL score, worsening IADL, MMSE score, years of education, incident stroke and log total volume of white matter changes as independent variables. Baseline history of depression, QoL and worsening IADL were significantly and independently associated with depression at 1 year. The association for $\log$ total volume of white matter changes did not quite reach statistical significance $(\mathrm{OR}=1.63, P=0.1)$.

\section{DISCUSSION}

\section{Main findings}

The principal finding of this longitudinal study was that severity of baseline white 
matter changes predicted subsequent depressive symptoms at 1 year, even after controlling for baseline depressive scores, QoL, worsening disability, incident stroke, educational level and MMSE score.

Baseline white matter changes did not, however, significantly predict depressive episodes over the following year. The fact that we were unable to demonstrate that age-related change in white matter volume was a significant independent predictor of this clinically important measure might have been because of a lack of power in this study to detect such an effect. Incident depression may have been underreported because of the method used to screen for mood disorders. This is supported by the relatively low number of participants with incident depression (85) over the year and the fact that there was a trend towards significance $(P=0.1)$.

Finally we established that QoL, worsening IADL and baseline GDS were significant and independent predictors of depressive symptoms, whereas incident stroke, MMSE score or years of education were not.

\section{Previous studies}

Our results support the findings of crosssectional studies that white matter changes are of aetiological significance for depressive symptoms in older adults. Already a strong association between white matter changes and depressive symptoms has been established in both hospital patients (O'Brien et al, 1996) and those living in the community (Steffens et al, 1999; de Groot et al, 2000). White matter changes are also known to predict poorer outcome (O’Brien et al, 1998) and treatment response (Hickie et al, 1997) in elderly people with depression. Neuropathological study has shown deep white matter changes to have an ischaemic basis (Thomas et al, 2002) and it is thought that these changes represent a marker for vascular pathology in clinically important areas of the brain. Following on from these findings the vascular depression hypothesis has been proposed (Baldwin \& O’Brien, 2002; Alexopoulos, 2005), which states that disruption of fronto-striatal circuits (which reciprocally link prefrontal cortex to basal ganglia) by vascular changes predisposes, perpetuates or exacerbates depressive syndromes. Our study suggests that white matter changes are associated with the development of depressive symptoms in older adults and hence supports the vascular depression hypothesis.

Despite the convergence of evidence from cross-sectional studies, results from longitudinal studies remain unclear. In a large community population, the Cardiovascular Health Study (Steffens et al, 2002) found that small basal ganglia lesions and large cerebral cortical white matter changes at baseline predicted persistence of depressive symptoms; furthermore, subcortical white matter changes predicted worsening of depressive symptoms over time. Our data are in line with these results. However they are at variance with the findings of the study from the National Institute of Mental Health (NIMH; Taylor et al, 2003). In a cohort of 133 participants with severe depression, an increase in severity of white matter changes over 2 years, but not static baseline white matter change, predicted depressive outcome scores after controlling for baseline depressive symptoms (Taylor et al, 2003). We did not assess lesion progression; however, potential reasons for these discrepancies may be the different and highly selected population used in the smaller NIMH cohort.

Another longitudinal study, the PROSPER study (Versluis et al, 2006), rather surprisingly found that white matter changes were not related to baseline depressive features or development of depressive symptoms at follow-up. Furthermore, no association was found between progression of white matter changes and the progression of depressive symptoms. This is clearly in contrast to our results and those of other studies. Discrepancies with the current evidence may have been because of a relatively low total volume of white matter changes of participants in the PROSPER study (median $1.7 v .13 \mathrm{ml}$ in this study) and the low rate of participation of people with depressive symptoms (8\% with GDS score $>3 v .35 \%$ in this study).

\section{Nature of the relationship}

Our longitudinal results provide an important demonstration that white matter changes pre-date, and therefore may be causally related to, the development of depressive symptoms. We cannot, however, dismiss the possibility that depressive symptoms may also pre-date white matter changes. The relationship may be bidirectional, just as it is with vascular risk factors and depression in older adults (Thomas $e t$ al, 2004; Baldwin, 2005). It is known that baseline vascular burden independently predicts depression (Mast, 2004) and yet it is also clear from longitudinal studies that depression is an independent risk factor for later developing coronary artery disease (Pratt et al, 1996), stroke (Everson et al, 1998) and cardiac mortality post-myocardial infarction (Frasure-Smith et al, 1999). The relationship is likely to be complex, although we have helped to unravel one aspect.

Interestingly, the temporal association demonstrated remained significant even after controlling for baseline cognitive scores and worsening disability. This suggests that this effect is not simply mediated through disability or secondary to a psychological reaction to declining cognitive performance, as has also been suggested (Cahn et al, 1996; Paterniti et al, 2002).

\section{Strengths and limitations of the study}

The strengths of the LADIS Study are the large number of community-based participants without significant disability and its multicentre design. Further strengths include the central measurement of all scans by a single operator and the use of a robust measure of the volume of age-related white matter changes which is less operator dependent and less susceptible to ceiling effects (van Straaten et al, 2006). Finally, a strong model for logistic analysis was constructed controlling for many potential confounders, including putative mediators of depressive features such as cognition, QoL, incident stroke and worsening disability.

Limitations include the recruitment of only those with white matter changes: consequently people with minimal or no health problems might have been excluded (although evidence suggests that up to $95 \%$ of older adults have some white matter changes; de Leeuw et al, 2001). Another potential limitation arises from the fact that some symptoms measured by the GDS, such as apathy and withdrawal, are not exclusive to a depressive syndrome and may also be manifestations of a mild cognitive dysexecutive syndrome. Despite this weakness we were able to demonstrate a significant association between white matter changes and depressive symptoms not only after controlling for cognitive confounders but also at higher GDS scores, when the symptoms are more likely to 
reflect a depressive syndrome. Finally, our results might have been influenced by sampling bias as the study population represents a heterogeneous group with a variety of different complaints but without significant disability. However, such bias is unavoidable in large multicentre studies and this heterogeneity might actually increase the generalisability of our findings.

\section{Clinical implications}

The most important implications concern the prevention of late-life depressive symptoms. These extend more widely to the elderly population as a whole because most participants had less disability and less depression than seen routinely in clinical practice. Recent evidence shows that treatment of hypertension slows progression of white matter changes (Schiffrin, 2005). We would therefore advocate tighter control of vascular risk factors to prevent the development of late-life depressive symptoms.

The presence of white matter changes on MRI, if considered alone, is of little clinical value in predicting future depressive symptoms. However, when white matter changes are taken along with other independent predictors such as quality of life and previous depressive episodes, they raise the index of suspicion for further depressive symptoms. Thus in clinical practice it is wise to take account of the presence of white matter changes on MRI when attempting to predict future depressive symptoms, and this might influence decisions regarding the frequency of clinical monitoring and the need for prophylactic antidepressants.

\section{Future research}

Large studies in a community population are indicated to establish whether static lesion volume, rate of progression, or both are the important determinants in predicting future depressive symptoms. Further research should also be directed at looking more precisely at the time course of emergence of changes and of mood symptoms, and whether modification of white matter changes influences the course of depressive symptoms.

\section{ACKNOWLEDGEMENTS}

The LADIS Study is supported by the European Union within the $\mathrm{V}$ European Framework Programme 'Quality of Life and Management of Living
Resources' (1998-2002), contract no. QLRT-200000446 as a concerted action.

\section{APPENDIX}

\section{List of participating centres and personnel}

Helsinki, Finland (Memory Research Unit, Department of Clinical Neurosciences, Helsinki University), Timo Erkinjuntti, MD, PhD, Tarja Pohjasvaara, MD, PhD, Pia Pihanen, MD, Raija Ylikoski, PhD, Hanna Jokinen, LPsych, Meija-Marjut Somerkoski, MPsych, Riitta Mäntylä, MD, PhD, Oili Salonen, MD, PhD; Graz, Austria (Department of Neurology and MRI Institute, Medical University Graz), Franz Fazekas, MD, Reinhold Schmidt, MD, Stefan Ropele, PhD, Brigitte Rous, MD, Katja Petrovic, MagPsychol, Ulrike Garmehi, Alexandra Seewann, MD; Lisbon, Portugal (Serviço de Neurologia, Centro de Estudos Egas Moniz, Hospital de Santa Maria), José M. Ferro, MD, PhD, Ana Verdelho, MD, Sofia Madureira, PsyD; Amsterdam, The Netherlands (Department of Radiology and Neurology, VU Medical Center), Philip Scheltens, MD, PhD, Ilse van Straaten, MD, Frederik Barkhof, MD, PhD, Alida Gouw, MD, Wiesje van der Flier, PhD; Gothenburg, Sweden (Institute of Clinical Neuroscience, Gothenburg University), Anders Wallin, MD, PhD, Michael Jonsson, MD, Karin Lind, MD, Arto Nordlund, PsyD, Sindre Rolstad, PsyD, Ingela Isblad, RN; Huddinge, Sweden (Karolinska Institute, Neurotec Department, Section of Clinical Geriatrics), Lars-Olof Wahlund, MD, PhD, Milita Crisby, MD, PhD, Anna Pettersson, Physiotherapist, Kaarina Amberla, PsyD; Paris, France (Department of Neurology, Hopital Lariboisiere), Hugues Chabriat, MD, PhD, Karen Hernandez, Psychologist, Annie Kurtz, Psychologist, Dominique Hervé, MD; Mannheim, Germany (Department of Neurology, University of Heidelberg, Klinikum Mannheim), Michael Hennerici, MD, Christian Blahak, MD, Hansjorg Baezner, MD, Martin Wiarda, PsyD, Susanne Seip, RN; Copenhagen, Denmark (Memory Disorders Research Group, Department of Neurology, Rigshospitalet, Copenhagen University Hospital, Denmark), Gunhild Waldemar, MD, DMSc, Egill Rostrup, MD, MSc, Charlotte Ryberg, MSc, Tim Dyrby MSc, Olaf B. Paulson, MD, DMSc; Newcastle upon Tyne, UK (Institute for Ageing and Health, University of Newcastle), John O'Brien, DM, Sanjeet Pakrasi, MRCPsych, Mani Krishnan MRCPsych, Andrew Teodorczuk, MRCPsych, Michael Firbank, PhD, Philip English, DCR. The Coordinating Centre is in Florence, Italy (Department of Neurological and Psychiatric Sciences, University of Florence), Domenico Inzitari, MD (Study Coordinator), Luciano Bartolini, PhD, Anna Maria Basile, MD, PhD, Eliana Magnani, MD, Monica Martini, MD, Mario Mascalchi, MD, PhD, Marco Moretti, MD, Leonardo Pantoni, MD, PhD, Anna Poggesi, MD, Giovanni Pracucci, MD, Emilia Salvadori, PhD, Michela Simoni, MD.

The LADIS Steering Committee comprises Domenico Inzitari, MD (Study Coordinator), Timo Erkinjuntti, MD, PhD, Philip Scheltens, MD, PhD, Marieke Visser, MD, PhD, Peter Langhorne, MD, BSC, PhD, FRCP who replaced Kjell Asplund, MD, $\mathrm{PhD}$ in 2005.

\section{REFERENCES}

Alexopoulos, G. S. (2005) Depression in the elderly. Lancet, 365, 1961-1970.

Baldwin, R. C. (2005) Is vascular depression a distinct subtype of depressive disorder? A review of the causal evidence. International Journal of Geriatric Psychiatry, 20, I-II.

Baldwin, R. C. \& O'Brien, J.T. (2002) Vascular basis of late-onset depressive disorder. British Journal of Psychiatry, 180, 157-160.

Cahn, D. A., Malloy, P. F., Salloway, S., et al (1996) Subcortical hyperintensities on MRI and activities of daily living in geriatric depression. Journal of Neuropsychiatry and Clinical Neurosciences, 8, 404-4II.

Chalmers, J., MacMahon, S., Mancia, G., et al (1999) World Health Organization International Society of Hypertension guidelines for the management of hypertension. Journal of Hypertension, 17, 151-183.

de Groot, J. C., de Leeuw, F. E., Oudkerk, M., et al (2000) Cerebral white matter lesions and depressive symptoms in elderly adults. Archives of General Psychiatry 57, $107 \mid-1076$.

de Leeuw, F. E., de Groot, J. C., Achten, E., et al (200I) Prevalence of cerebral white matter lesions in elderly people: a population based magnetic resonance imaging study. The Rotterdam Scan Study. Journal of Neurology, Neurosurgery and Psychiatry, 70, 9-14.

Euro-Qol Group (1990) Euro-Qol: a new facility for the measurement of health related quality of life. Health Policy, 16, 199-208.

Everson, S. A., Roberts, R. E., Goldberg, D. E., et al

(1998) Depressive symptoms and increased risk of stroke mortality over a 29-year period. Archives of Internal Medicine, 158, II33-||38.

Fazekas, F., Chawluk, J. B., Alavi, A., et al (1987) MR signal abnormalities at I.5 T in Alzheimer's dementia and normal aging. American journal of Neuroradiology, $\mathbf{8}$, $421-426$.

Firbank, M. J., O'Brien, J.T., Pakrasi, S., et al (2005) White matter hyperintensities and depression preliminary results from the LADIS study. International Journal of Geriatric Psychiatry, 20, 674-679.

Folstein, M. F., Folstein, S. E. \& McHugh, P. R. (1975) 'Mini-mental state'. A practical method for grading the cognitive state of patients for the clinician. Journal of Psychiatric Research, 12, 189-198.

Frasure-Smith, N., Lesperance, F., Juneau, M., et of (1999) Gender, depression, and one-year prognosis after myocardial infarction. Psychosomatic Medicine 6I, 26-37.

Gouw, A., Van der Flier, W., van Straaten, E., et a (2006) Simple versus complex assessment of white matter hyperintensities in relation to physical performance and cognition: the LADIS study. Journal of Neurology, 253, II89-1196.

Hatano, S. (1976) Experience from a multicentre stroke register: a preliminary report. Bulletin of the World Health Organization, 54, 54I-553.

Hickie, I., Scott, E., Wilhelm, K., et al (1997)

Subcortical hyperintensities on magnetic resonance imaging in patients with severe depression: a longitudinal evaluation. Biological Psychiatry, 42, 367-374.

Lawton, M. P. \& Brody, E. M. (1969) Assessment of older people: self maintaining and instrumental activities of daily living. Gerontologist, 9, 179-186.

Mast, B. T. (2004) Cerebrovascular disease and late-life depression: a latent-variable analysis of depressive 
symptoms after stroke. American Journal of Geriatric Psychiatry, 12, 315-322.

O'Brien, J., Desmond, P., Ames, D., et al (1996) A magnetic resonance imaging study of white matter lesions in depression and Alzheimer's disease. British Journal of Psychiatry, 168, 477-485.

O'Brien, J., Ames, D., Chiu, E., et al (1998) Severe deep white matter lesions and outcome in elderly patients with major depressive disorder: a follow-up study. BM/, 317, 982-984.

O'Brien, J., Firbank M. J., Krishnan, M. S., et al (2006) White matter hyperintensities rather than lacunar infarcts are associated with depressive symptoms in older people: The LADIS Study. American Journal of Geriatric Psychiatry, 14, 834-84I.

Pantoni, L., Basile, A., Pracucci, G., et al (2005) Impact of age-related cerebral white matter on the transition to disability - the LADIS study: rationale, design and methodology. Neuroepidemiology, 24, 51-62.

Paterniti, S., Verdier-Taillefer, M-H., Dufouil, C., et a (2002) Depressive symptoms and cognitive decline in elderly people: longitudinal study. British Journal of Psychiatry, I8I, 406-410.

Pratt, L. A., Ford, D. E., Crum, R. M., et al (1996) Depression, psychotropic medication, and risk of myocardial infarction. Prospective data from the Baltimore ECA follow-up. Circulation, 15, 3123-3129.

Schiffrin, E. (2005) Blood pressure lowering in PROGRESS (Perindopril Protection Against Recurrent Stroke Study) and white matter hyperintensities: should this progress matter to patients? Circulation, II2, 15251526.

Steffens, D. C., Helms, M. J., Krishnan, K. R., et a (1999) Cerebrovascular disease and depression symptoms in the cardiovascular health study. Stroke, $\mathbf{3 0}$, 2159-2166.

Steffens, D. C., Krishnan, K. R. R., Crump, C., et a (2002) Cerebrovascular disease and evolution of

ANDREW TEODORCZUK, MRCPsych, JOHN T. O'BRIEN, DM, MICHAEL J. FIRBANK, PhD, Institute for Ageing and Health, University of Newcastle uponTyne, UK; LEONARDO PANTONI, MD, ANNA POGGESI, MD, Department of Neurological and Psychiatric Sciences, University of Florence, Italy; TIMO ERKINJUNTTI, MD, Memory Research Unit, Department of Neurology, University of Helsinki, Finland; ANDERS WALLIN, MD, Institute of Clinical Neuroscience, Gothenburg University, Sweden; LARS-OLOF WAHLUND, MD, Karolinska University Hospital, Huddinge, Sweden; ALIDA GOUW, MD, Alzheimer Centre and Department of Neurology, VU University Hospital, Amsterdam, The Netherlands; GUNHILD WALDEMAR, MD, Memory Disorders Research Group, Department of Neurology, Rigshospitalet, Copenhagen University Hospital, Denmark; REINHOLD SCHMIDT, MD, Department of Neurology and MRI Institute, Medical University Graz, Austria; JOSÉ M. FERRO, MD, Serviço de Neurologia, Centro de Estudos Egas Moniz, Hospital de Santa Maria, Lisboa, Portugal; HUGUES CHABRIAT, MD, Department of Neurology, Hopital Lariboisiere, Paris, France; HANSJÖRG BÄZNER, MD, Department of Neurology, University of Heidelberg, Universitätsklinikum Mannheim, Germany; DOMENICO INZITARI, MD, Department of Neurological and Psychiatric Sciences, University of Florence, Italy

Correspondence: DrTeodorczuk, Institute for Ageing and Health, Newcastle General Hospital,Westgate Road, Newcastle uponTyne NE4 6BE, UK. Email: andrew.teodorczuk@ncl.ac.uk

(First received 9 February 2007, final revision 26 April 2007, accepted 27 June 2007)

depressive symptoms in the Cardiovascular Health Study. Stroke, 33, 1636-1644.

Taylor, W. D., Steffens, D. C., MacFall, J. R., et al (2003) White matter hyperintensity progression and late-life depression outcomes. Archives of General Psychiatry, 60, 1090-1096.

Thomas, A. J., O'Brien, J.T. \& Davis, S. (2002) Ischemic basis for deep white matter hyperintensities in major depression - a neuropathological study. Archives of General Psychiatry, 59, 785-792.

Thomas, A. J., Kalaria, R. \& O'Brien, J.T. (2004) Depression and vascular disease: what is the relationship? Journal of Affective Disorders, 79, 8I-95. van Straaten, E. C., Fazekas, F., Rostrup, E., et al (2006) Impact of white matter hyperintensities scoring method on correlations with clinical cata: the LADIS Study. Stroke, 37, 836-840.

Versluis, C. E., van der Mast, R. C., van Buchem, M. A., et al (2006) Progression of cerebral white matter lesions is not associated with development of depressive symptoms in elderly subjects at risk of cardiovascular disease. The PROSPER Study. International journal of Geriatric Psychiatry, 2I, 375-38

Yesavage, J. A. (1988) Geriatric Depression Scale. Psychopharmacology Bulletin, 24, 709-7II. 